\title{
Virtual Reality-Enhanced Ultrasound Guidance for Atrial Ablation: In vitro Epicardial Study
}

\author{
Cristian A. Linte ${ }^{1,2}$, Andrew Wiles ${ }^{1,3}$, John Moore $^{1}$, \\ Chris Wedlake ${ }^{1}$, and Terry M. Peters ${ }^{1,2,3}$ \\ ${ }^{1}$ Imaging Research Laboratories, Robarts Research Institute \\ ${ }^{2}$ Biomedical Engineering Graduate Program, University of Western Ontario \\ ${ }^{3}$ Department of Medical Biophysics, University of Western Ontario, \\ London, ON, Canada
}

\begin{abstract}
In an effort to reduce morbidity of cardiac interventions, minimizing invasiveness inevitably leads to limited visual access to the surgical targets. To address these limitations, we provide the surgeons with a robust visualization environment that integrates interventional ultrasound imaging augmented with pre-operative anatomical models and virtual surgical instruments within a virtual reality environment. Here we present an in vitro study on a cardiac phantom that mimics an ablation therapy procedure, which allows us to assess the feasibility of our surgical system in comparison to traditional intra-operative ultrasound imaging. Following surgical target identification via an electro-anatomical model, the "ablation procedure" is performed blindly. A $2.8 \mathrm{~mm}$ RMS targeting error is achieved using our novel surgical system. This level of accuracy is adequate from both a clinical and engineering perspective, under the inherent procedure requirements and limitations of the system.
\end{abstract}

\section{Introduction}

Despite pervasive use of medical imaging for therapy guidance, the progress towards off-pump intracardiac interventions has been hampered by the lack of sufficient visualization and surgical guidance. For the case of atrial fibrillation thepary (AF) for instance, the highest curative rates are achieved by surgery, however at the expense of highly invasive approaches.

Ablation catheters are used instead to either "burn" or "freeze" the tissue by means of high-frequency energy delivery under fluoroscopy guidance, creating lesions that isolate the arrhythmia foci [1. Nevertheless, several disadvantages have been identified with respect to x-ray fluoroscopy and its use for catheter guidance: first multiple views are required to localize the catheter tip in 3D space, leading to significant radiation exposure; second, due to their low soft tissue contrast, X-ray images lack anatomical context; last, the clinician has to mentally fuse the electrical activation maps with the anatomical information provided via fluoroscopy to identify the optimal surgical path.

Several approaches to minimally invasive ablation guidance have evolved, employing intracardiac echocardiography [2, interventional magnetic resonance 
imaging (MRI) [3] or MR - fluoroscopy guidance [4] for intra-operative visualization. However, none of these techniques show any attempt of fusing the pre-operative planning stage with the intra-operative therapy delivery.

To address these limitations, we have developed a novel surgical guidance system to facilitate minimally invasive beating heart therapy. Our surgical platform relies on trans-esophageal echocardiography (TEE) for real-time guidance, preoperative cardiac models for anatomical enhancement [5], and magnetic tracking technology for instrument tracking [7. In the context of ablation therapy, virtual reality (VR)-enhanced ultrasound (US) guidance eliminates the risks associated with fluoroscopic imaging and enables the mapping of dynamic EP data onto the pre-operative cardiac models [8. Ultimately, clinicians can explore the intracardiac environment using registered pre-operative models as guides, and navigate surgical tools intrinsically relative to cardiac anatomy.

\section{Methodology}

This paper focuses on assessing the VR-enhanced US surgical platform for procedures that resemble ablation therapy in absence of direct vision. As the overall goal is to employ this novel interventional tool in guiding intracardiac procedures, we present an in vitro study conducted on a cardiac phantom (Fig. 1), where all experiments mimic a blinded epicardial intervention. Surgical targets can be identified using electro-anatomical models by mapping the EP data onto the pre-operative cardiac model. Therapy consists of navigating a tracked pointer to a specific target under VR-enhanced US guidance, and interventional success is measured via targeting accuracy and procedure time.
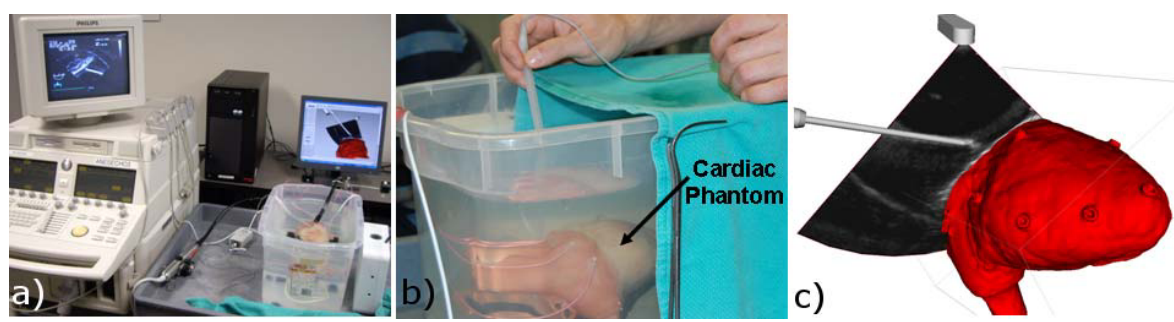

Fig. 1. a) Experimental setup including US scanner, tracking system and cardiac phantom; b) Phantom submerged in water and blinded from the user; c) Virtual reality environment showing cardiac model, pointer tool, US probe and image fan

\subsection{Pre-operative Stage}

Image Acquisition and Modeling: We employed a realistic heart phantom (The Chamberlain Group, Great Barrington, MA, USA) that exhibits similar characteristics to both human and porcine hearts, with a highly detailed exterior, as well as comparable size and anatomy (Fig. 2). Ten CT-visible fiducial 

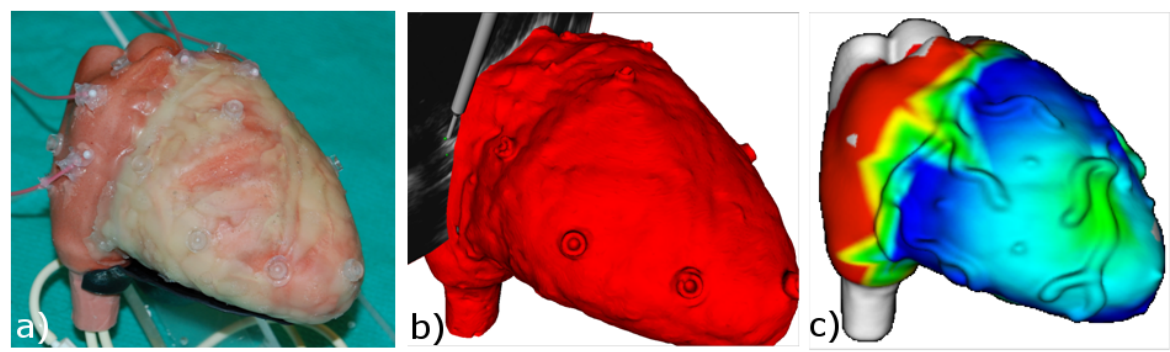

Fig. 2. a) Cardiac phantom used for in vitro experiments; b) Surface model of the phantom segmented from the CT image; c) Electro-anatomical model obtained by mapping EP data onto a pre-operative model to serve with surgical target identification.

markers were attached on the epicardial surface of the phantom and a computer tomography $(\mathrm{CT})$ scan was acquired. A pre-operative model was generated from the CT volume using automatic segmentation tools available within the Vascular Modeling ToolKit1 (Fig. 2).

EP Study and Diagnosis: We previously demonstrated the extension of 3D static cardiac mapping environment into one that takes full advantage of $4 \mathrm{D}$ pre-operative modeling [8]. Recorded EP data can be encoded according to their spatial and temporal time-stamps, as prescribed by the tracking system and ECG-gating, respectively. As such, 4D mapping enables the augmentation of the pre-operative models [9] with functional data, leading to electro-anatomical models [10] suitable for both surgical planning and intra-procedure guidance.

In this study we used EP data acquired in an in vivo porcine study from the epicardial surface of the left and right atria under induced AF. An electroanatomical model was obtained by mapping the EP data onto the pre-operative epicardial phantom model (Fig. 2).

Surgical Target Identification: Using the superior display provided by the electro-anatomical model, four small regions on the epicardial surface model, designated as potential arrhythmia foci, were selected as surgical targets for our in vitro study. These targets were physically labeled onto the phantom surface by means of $3.2 \mathrm{~mm}$ Teflon spheres, visible under both CT and US. To enable quantitative assessment of therapy guidance, each Teflon sphere was tracked using a 5 degree-of-freedom (DOF) magnetic tracking sensor. Ultimately, a second pre-operative CT scan of the phantom was acquired and a new surface model was generated, displaying the pre-operative location of the surgical targets.

\section{$2.2 \quad$ Intra-procedure Stage}

Intra-operative Equipment and Setup: All experiments were performed in our image-guided surgery laboratory. The cardiac phantom was submerged in a

\footnotetext{
${ }^{1}$ Vascular Modeling Toolkit:

http://villacamozzi.marionegri.it/ luca/vmtk/doku.php
} 
water bath to enable US image acquisition. Virtual representations of the US transducer and tracked pointer tool, accompanied by the pre-operative model of the cardiac phantom were registered to their physical counterparts and displayed as part of the VR environment.

Intra-procedure US images were acquired on a Philips SONOS 7500 scanner using an adult 2D TEE transducer, tracked using the NDI Aurora magnetic tracking system (MTS). In addition to the tracked TEE probe, a virtual representation of the tracked pointer was also included in the VR environment.

Therapy Delivery and Surgical Guidance: During ablation therapy, surgeons face the challenge of navigating an ablation tool to a specific surgical site. We have mimicked a similar surgical task for our in vitro experiments: each user blindly navigates the tracked pointer — the surgical instrument, to the Teflon spheres - the surgical targets. Once on target, the positions of both the surgical target and the pointer tip are acquired. The blind procedure mimics a minimally invasive, closed-chest intervention, where surgeons would not have access to direct target visualization. Rather, the users rely on either US imaging or VR-enhanced US guidance to assist them with both the navigation towards target and on-target positioning of the tracked tool.
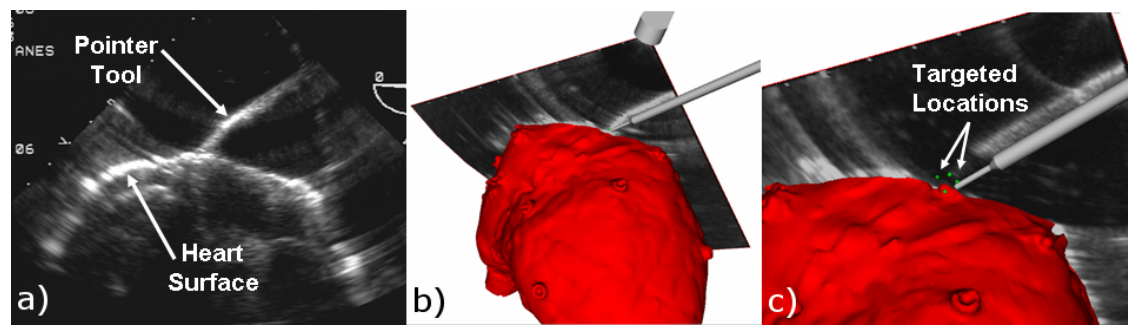

Fig. 3. a) Typical image acquired under US image guidance showing poor 3D perception of pointer tool; b) VR-enhanced US guidance display showing pre-operative cardiac model, virtual model of surgical tool, TEE probe and US fan; c) "Therapy delivery" under VR-enhanced US guidance: "true surgical target" surrounded by "ablated" sites

US Image Guidance: Intra-operative visualization was first provided via US imaging, upon which users relied to both navigate the surgical tool to the target and also to position the tool tip onto the surgical target. Given the 2D images, the navigation to target was limited by the lack of anatomical context, and capabilities of the images to portray the 3D scene (Fig. 3). Significant difficulties were encountered when neither the target, nor the surgical tool were visible in the 2D US image plane.

VR-Enhanced US Guidance: Enhanced visualization was achieved by integrating both the pre- and intra-operative information in a far superior display, where the 2D US images that previously lacked context and offered a poor perception of the surgical tools were augmented with the pre-operative anatomical models 
(Fig. 3) and virtual representations of the surgical tools. Using VR-enhanced US guidance, users had access to sufficient anatomical context to accurately navigate the tracked tool to the target, and in the event of slight mis-registrations between the model and its physical counterpart, the real-time information supplied via US imaging is crucial during therapy delivery.

\section{Procedure Assessment and Results}

Eight users ( 4 experts and 4 novices) conducted the in vitro ablation procedure on four surgical targets. Each target was attempted 3 times by each user under each guidance modality (US image guidance and VR-enhanced US guidance). The procedure outcome was assessed quantitatively, according to the accuracy and duration of each "therapeutic" trial. We performed an analysis of variance (ANOVA) followed by a Bonferroni post-test (GraphPad Prism 4.0) to test significant differences in the procedure accuracy and duration with respect to the level of expertise of the users and the guidance modality employed. We also assessed the interventional system qualitatively, via a brief survey.

\subsection{Quantitative Assessment: Accuracy and Duration}

Guidance accuracy was computed as the distance between the centre of the surgical target (tracked Teflon sphere) and centre of the tip of the surgical instrument (pointer tool), where their positions were reported by the MTS. The duration of the procedure was measured as the time elapsed during each trial. Table 1 summarizes the distance errors and procedure time, according to the users' expertise and guidance modality employed.

Table 1. Summary of guidance accuracy (RMS distance error) and procedure time (Mean \pm Std. Error) reported according to user's expertise and guidance modality

\begin{tabular}{|c||c|c|c|c|}
\hline Guidance & \multicolumn{2}{|c|}{ US Imaging } & \multicolumn{2}{c|}{ VR-enhanced US } \\
\hline \hline Distance Error $(\mathrm{mm})$ & \multicolumn{2}{|c|}{$3.38 \pm 0.28$} & \multicolumn{2}{|c|}{$2.58 \pm 0.12$} \\
RMS Error $(\mathrm{mm})$ & \multicolumn{2}{|c|}{4.37} & \multicolumn{2}{|c|}{2.83} \\
Elapsed Time $(\mathrm{sec})$ & \multicolumn{2}{|c|}{$10.09 \pm 0.64$} & \multicolumn{2}{c|}{$15.07 \pm 1.25$} \\
\hline \hline Expertise & Experts & Novice & Experts & Novice \\
\hline \hline Distance Error $(\mathrm{mm})$ & $3.10 \pm 0.36$ & $3.67 \pm 0.42$ & $2.63 \pm 0.16$ & $2.54 \pm 0.17$ \\
RMS Error (mm) & 4.01 & 4.72 & 2.86 & 2.77 \\
Elapsed Time (sec) & $7.73 \pm 0.62$ & $9.92 \pm 0.76$ & $12.55 \pm 0.98$ & $20.11 \pm 1.43$ \\
\hline
\end{tabular}

These results show improved procedure accuracy and more consistent targeting (Fig. 5) under VR-US guidance. While the expert users achieved better targeting under US imaging and a slightly shorter trial time compared to the novices, the novice group on the other hand, experienced a dramatic improvement in accuracy when employing the new guidance system, however at the expense of slightly longer durations. 

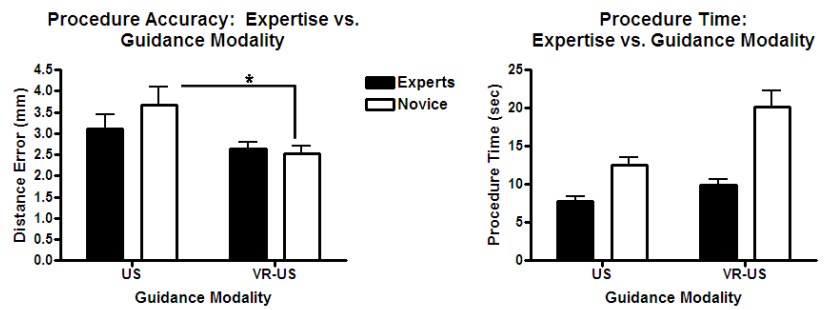

Fig. 4. Procedure accuracy and duration achieved by the expert and novice groups under both guidance modalities. Note that significant targeting accuracy improvement $(* p<0.001)$ was achieved by the novice group under VR-US vs. US image guidance.

\subsection{Qualitative Assessment: System Versatility}

Each user completed a survey where they ranked the performance of the interventional system and its clinical relevance one a scale from 1 (poor) to 5 (highly effective) according to the criteria reported in Table 2. Both user groups appreciated the superior VR-US augmented display, which contributed to increased guidance intuitiveness and targeting confidence, both clinically relevant.

Table 2. Qualitative Surgical Guidance Assessment: Summary Table

\begin{tabular}{|c||c|c|c|c|}
\hline \multicolumn{1}{|c||}{ Evaluation } & \multicolumn{2}{c|}{ Experts } & \multicolumn{2}{c|}{ Novice } \\
\cline { 2 - 5 } Criteria & US & VR-US & US & VR-US \\
\hline \hline Display Quality & 1.75 & 4.5 & 2.25 & 3.75 \\
Intuitiveness & 1.75 & 4.25 & 1.75 & 4.5 \\
User's Confidence & 2.5 & 4.75 & 2.25 & 4.25 \\
Clinical Relevance & 4.0 & 4.75 & 3.5 & 4.75 \\
\hline
\end{tabular}

\section{Discussion}

The VR-enhanced US guidance led to more accurate targeting compared to the US guidance alone, as confirmed by the statistical significance tests $(p<0.05)$. Nevertheless, targeting accuracy of both experts and novice users was comparable and not significantly different $(p>0.05)$ under each guidance modality (Fig. 4). While experts achieved a better targeting accuracy under US image guidance compared to the novice group (due to their extensive exposure to interventional US), we do notice a highly significant $(p<0.001)$ accuracy improvement for the novice group when using VR-enhanced US guidance. Hence, for this group, the VR-US guidance facilitated the tool-to-target navigation, while the inadequate visualization, lack of 3D perspective, and limited US image quality contributed to severe difficulties in surgical navigation encountered under sole US imaging.

The procedure duration suggested that VR-US guidance leads to slightly longer times than US image guidance for both experts and novices. Moreover, 
Expert Group - Targeting Distribution:

US vs VR-US Guidance

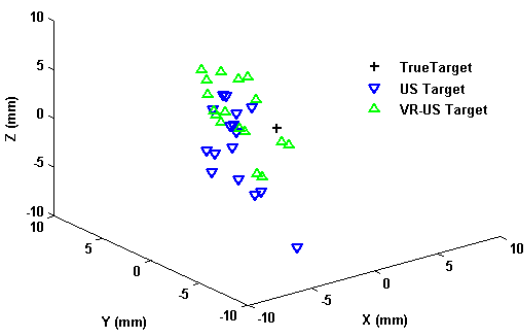

Novice Group - Targeting Distribution: US vs. VR-US Guidance

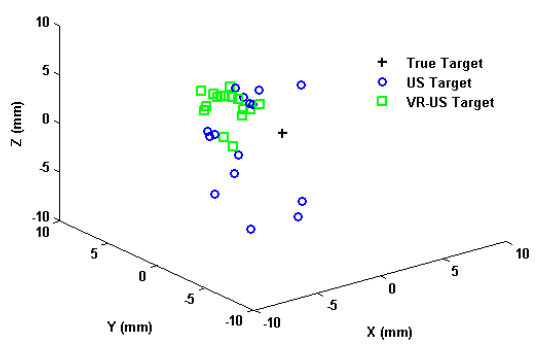

Fig. 5. Distribution of targeted locations around the true target achieved using both guidance modalities. Note that VR-US guidance led to more consistent targeting.

the expert group shows significantly shorter trial times compared to the novice group under both VR-US guidance $(p<0.001)$ and US guidance $(p<0.05)$. Considering that experts are highly accustomed to interventional US, the use of the hybrid VR-US system led to longer procedure times $(p<0.05)$, however resulting in more accurate targeting. For the novice group, although the procedure times were somewhat higher under VR-US guidance, the novel guidance method led to significantly higher targeting accuracy $(p<0.001)$. Considering that neither group has previously employed VR-based guidance, we therefore expect that procedure times will improve with adequate training.

From a clinical perspective and considering that most ablation procedures are performed along a path rather than on a specifically sized target, the $2.83 \mathrm{~mm}$ RMS error achieved under VR-enhanced US guidance is reasonable. From an engineering perspective, the error achieved is also acceptable given the accuracy of the magnetic tracking on the order of $1-2 \mathrm{~mm}$.

The true benefits of integrating virtual models within the visualization environment become transparent in a dynamic environment. We expanded this work into a dynamic study: an ECG-gated CT dataset of the beating heart phantom was acquired and post-processed to generate a dynamic pre-operative model, the locations of the surgical targets were identified in the planning stage, and their true positions were supplied intra-operatively by the MTS. Selected users attempted dynamic targeting under both guidance modalities and preliminary results have shown a consistent trend towards improved targeting accuracy and procedure time achieved under VR-US guidance vs. sole US imaging.

Moreover, while our surgical targets (Teflon spheres) were identified with sufficient ease under US imaging, true surgical targets on the heart wall cannot be easily distinguished from the surroundings. Therefore, virtual anatomical models that surgeons can use for both pre-operative planning and intraprocedure guidance do provide crucial information that facilitates intra-operative navigation. 


\section{Conclusions}

We assessed the feasibility of a novel VR-enhanced US guidance system in the context of an in vitro epicardial ablation therapy. We show that targeting accuracy was improved under VR-US guidance compared to US image guidance across all users, and furthermore, the novices achieved higher and more consistent targeting accuracy than the experts when using VR-US guidance.

\section{References}

1. Kottkamp, H., Hindricks, G., Autschbach, R., et al.: Specific linear left atrial lesions in atrial fibrillation: Intraoperative radiofrequency ablation using minimally invasive surgical techniques. J. Am. Coll. Cardiol. 40, 475-480 (2002)

2. Chu, E., Fitzpartick, A., Chin, M., et al.: Radiofrequency catheter ablation guided by intracardiac eachcardiography. Circulation 89, 1301-1305 (1994)

3. Lardo, A.C., McVeigh, E., Halperin, H., et al.: Visualization and temporal/spatial characterization of cardiac radiofrequency ablation lesions using magnetic resonance imaging. Circulation 102, 698-705 (2000)

4. Razavi, R., Hill, D.L.G., Keevil, S.F., et al.: Cardiac catheterization guided by MRI in children and adults with congenital heart disease. Lancer 362, 1877-1882 (2003)

5. Wierzbicki, M., Drangova, M., Guiraudon, G.M., Peters, T.M.: Validation of dynamic heart models obtained using non-linear registration for virtual reality training, planning, and guidance of minimally invasive cardiac surgeries. Med. Image Anal. 8, 387-401 (2004)

6. Linte, C.A., Wierzbicki, M., Moore, J., et al.: Towards subject-specific models of the dynamic heart for mitral valve surgery. In: Ayache, N., Ourselin, S., Maeder, A. (eds.) MICCAI 2007, Part II. LNCS, vol. 4792, pp. 94-101. Springer, Heidelberg (2007)

7. Linte, C.A., Moore, J., Wiles, A.D., et al.: Virtual reality-enhanced ultrasound guidance: A novel technique for intracardiac interventions. Comput. Aided Surg. 13, 82-94 (2008)

8. Wilson, K., Guiraudon, G., Jones, D., Peters, T.M.: 4D shape registration for dynamic electrophysiological cardiac mapping. In: Larsen, R., Nielsen, M., Sporring, J. (eds.) MICCAI 2006. LNCS, vol. 4191, pp. 520-526. Springer, Heidelberg (2006)

9. Linte, C.A., Wierzbicki, M., Moore, J., et al.: On enhancing planning and navigation of beating-heart mitral valve surgery using pre-operative cardiac models. Proc. of IEEE Eng. Med. Biol. Soc., 475-78 (2007)

10. Wilson, K., Jones, D.L., Linte, C.A., et al.: Dynamic cardiac mapping on patientspecific models. LNCS, TBA (2008) 